\title{
Self-similarity approach for prediction and analysis of experiments at the accelerator complex NICA
}

\author{
Elina G. Baldina ${ }^{1,2, *}$ and Anton A. Baldin ${ }^{1,2}$ \\ ${ }^{1}$ Joint Institute for Nuclear Research, Dubna, 141980 Russia \\ ${ }^{2}$ Institute for Advanced Studies "OMEGA", Dubna, 141980 Russia
}

\begin{abstract}
The given functional self-similarity solution quantitatively describes angular, energy and A-dependences of inclusive production cross sections for hadrons in relativistic nuclear collisions. It is applied to quantitative estimation of $\mathrm{D}$, as well as $\varphi$ and $\mathrm{J} / \psi$ meson production in collider experiment at the NICA accelerator complex with heavy nuclei. The results can be used for optimization of kinematically registered parameters for investigation of collective phenomena.
\end{abstract}

Self-similarity is a special symmetry of solutions when a change in scales of independent variables is compensated by a self-similarity transformation of other dynamical variables.

The main idea of application of the self-similarity approach in physics is to reduce the number of variables in a physical law.

The self-similarity solution $[1,2]$ has proved to describe well the wide set of experimental data, including the cumulative, subthreshold particle production [3-5]. It was applied for prediction of $\varphi$ and $\mathrm{J} / \psi$ meson production in heavy-ion collisions [6]. Here, we apply it for estimation of charm production at the future NICA collider.

Particles containing strange and charm quarks are important probes of the excited medium created in heavy-ion collisions [7]. Experimental data on open and hidden charm in heavy-ion collisions at low and intermediate energies are absent. Therefore, it is important to study the processes of charm production near threshold energies. Particles containing charm quarks are believed to be produced at the first stage of the reaction, thus allowing one to examine the evolution of the degrees-of-freedom in a collision.

Below we present the calculated invariant cross sections for production of the lightest charmed particles, $\mathrm{D}+$, D- mesons.

Fig. 1 shows the invariant cross section of the $\mathrm{D}$ meson production at $0^{\circ}$ and $10 \mathrm{MeV}$ in the collider geometry in $\mathrm{Au}-\mathrm{Au}$ collisions as a function of $\mathrm{S}^{1 / 2}$. The inset shows in more detail the NICA range for heavy ions, $\mathrm{S}^{1 / 2}=4-11 \mathrm{GeV}$. It can be seen that NICA covers the most interesting region of charm production growth, and the experimental study in this range can contribute to the understanding of QCD physics.

*e-mail: e.baldina@mail.ru 


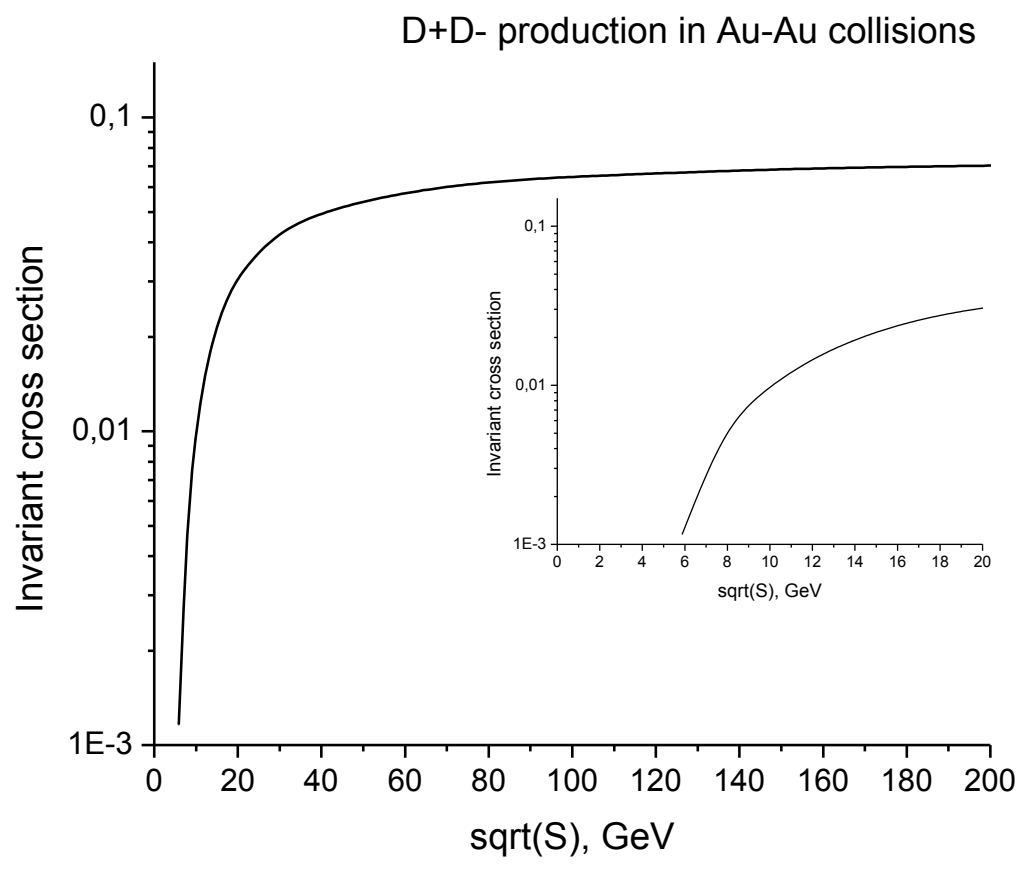

Figure 1a. Invariant cross section $\left(\mathrm{mb} \cdot \mathrm{GeV}^{-2} \cdot \mathrm{c}^{3} \cdot \mathrm{sr}^{-1}\right)$ of the $\mathrm{D}+\mathrm{D}$ - meson production at $0^{\circ}$ and 10 $\mathrm{MeV}$ in $\mathrm{Au}-\mathrm{Au}$ collisions as a function of $\mathrm{S}^{1 / 2}$

It can also be seen that the expected cross section values (Fig. 1b) are above $10^{-7}$ in the NICA momentum range, which is experimentally measurable at the planned NICA facilities.

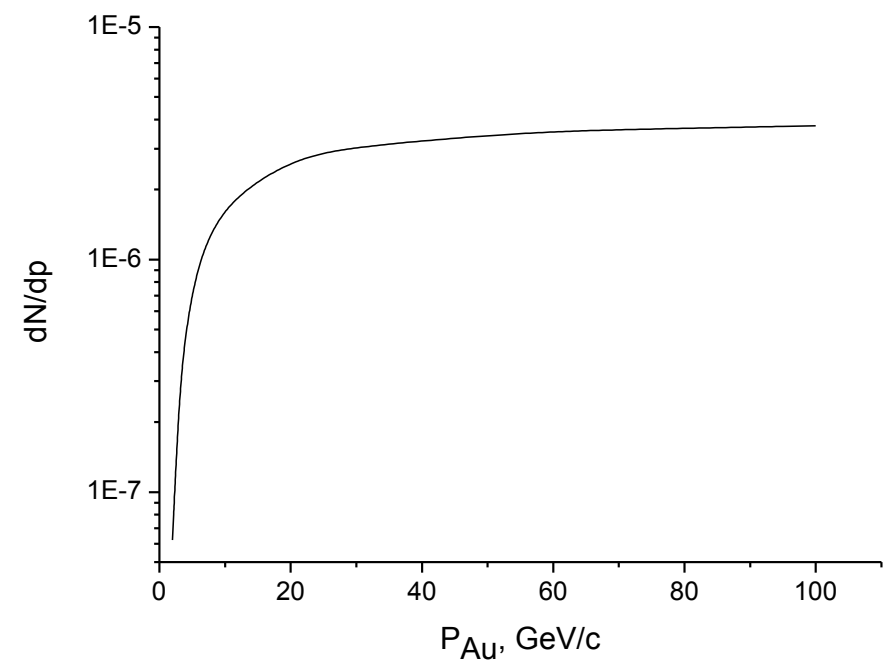

Figure 1b. Cross section of the $\mathrm{D}+\mathrm{D}-$ meson production at $0^{\circ}$ and $10 \mathrm{MeV}$ in $\mathrm{Au}-\mathrm{Au}$ collisions as a function of momentum of colliding particles 
Fig. 2 illustrates the D meson production at zero angle with increasing collision energy of heavy Au ions. The range achievable at NICA covers particle momenta of up to $6-8 \mathrm{GeV} / \mathrm{c}$ with an invariant cross section level of about 10-4 at small angles and about $2 \mathrm{GeV} / \mathrm{c}$ at large angles. It should be noted that the particle production probability strongly depends on the angle for the collider geometry, as well as the fixed target geometry. This presents difficulties in simulations because particle generators usually do not take this into account.

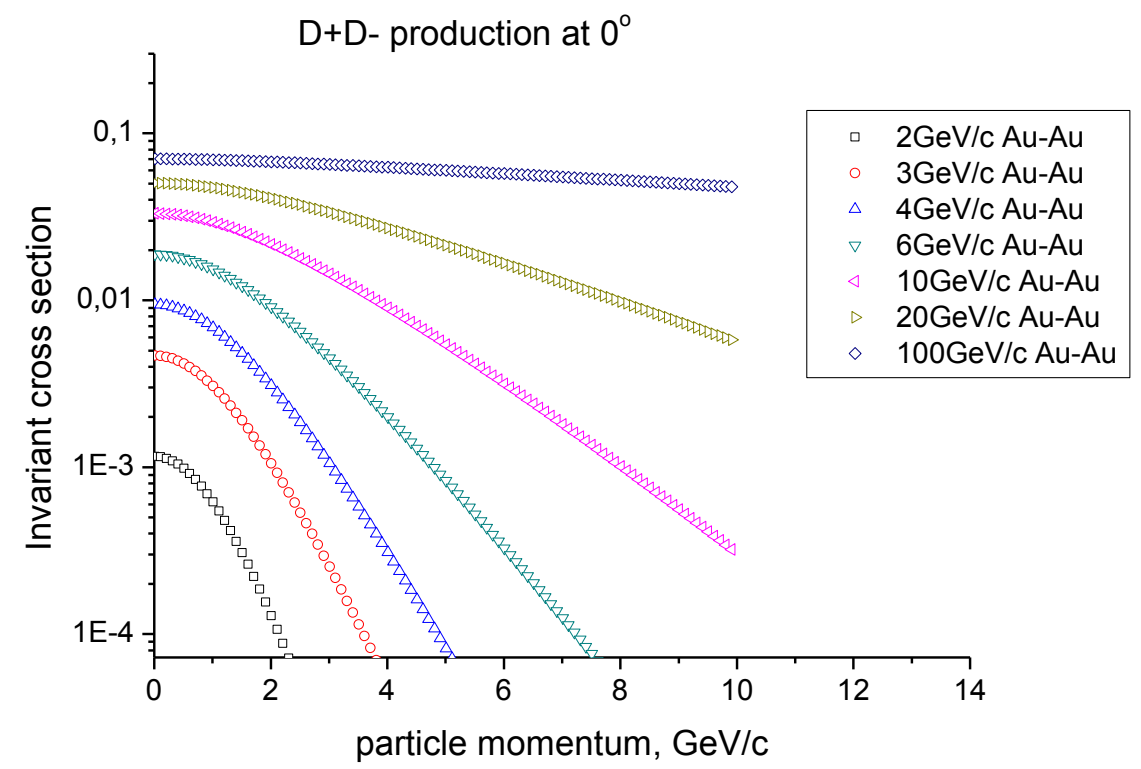

Figure 2. D meson production at zero angle in Au-Au collisions for different collision energies (invariant cross section in $\mathrm{mb} \cdot \mathrm{GeV}^{-2} \cdot \mathrm{c}^{3} \cdot \mathrm{sr}^{-1}$ )

Let us study the cross section behavior for different D meson momenta and angles in the laboratory frame.

Fig. 3 shows the cross section of D meson production in Au-Au collisions at $\sqrt{S}=9.875$ $\mathrm{GeV}\left(\mathrm{P}_{\mathrm{Au}}=4 \mathrm{GeV} / \mathrm{c}\right)$ for different $\mathrm{D}$ momenta. 


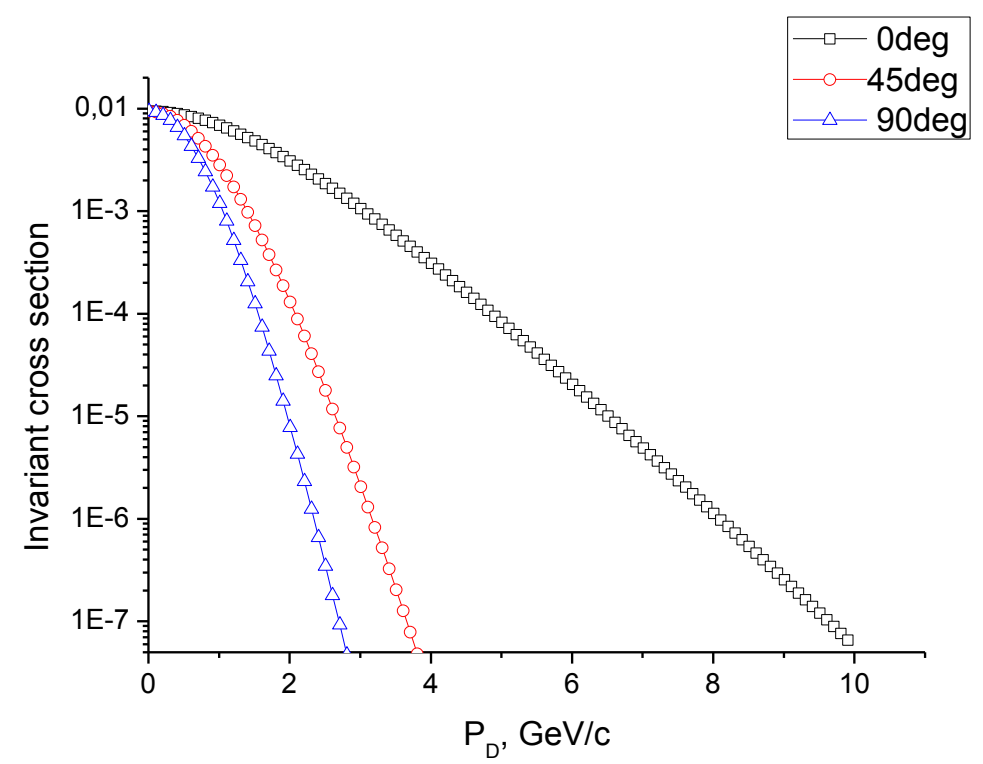

Figure 3. Invariant cross section $\left(\mathrm{mb} \cdot \mathrm{GeV}^{-2} \cdot \mathrm{c}^{3} \cdot \mathrm{sr}^{-1}\right)$ of the $\mathrm{D}+\mathrm{D}$ - production in $\mathrm{Au}-\mathrm{Au}$ collisions at $4 \mathrm{GeV} / \mathrm{c}$ for 0,45 , and $90^{\circ}$

It can be reasonably expected that particles are preferably produced near the plane of symmetry of the reaction (see Figs. 3, 4). The farther from the plane of symmetry, the sharper the cross section decreases with increasing particle momentum. Thus, for a registration angle of $45^{\circ}$ in laboratory frame, an invariant cross section of a level of $10-4 \mathrm{mb} \cdot \mathrm{GeV}-2 \cdot \mathrm{c} 3 \cdot \mathrm{sr}-1$ and higher can be expected for D momenta of up to $2 \mathrm{GeV} / \mathrm{c}$. For an angle of $90^{\circ}$, this cross section level is achieved for less energetic particles, up to about $1.5 \mathrm{GeV} / \mathrm{c}$. While at $0^{\circ}, \mathrm{D}$ mesons with a momentum of nearly $5 \mathrm{GeV} / \mathrm{c}$ can be measured for the above cross section level (see Fig. 3).

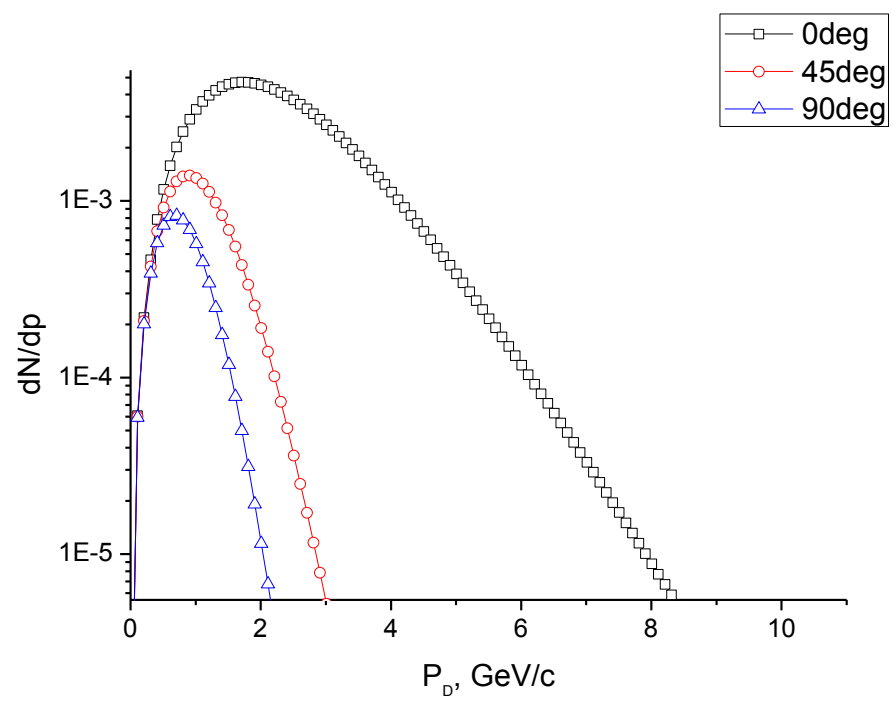

Figure 4. $\mathrm{dN} / \mathrm{dp}$ for $\mathrm{D}+\mathrm{D}$ - production as a function of $\mathrm{D}$ meson momentum in $\mathrm{Au}-\mathrm{Au}$ collisions at $4 \mathrm{GeV} / \mathrm{c}(\sqrt{S}=9.875 \mathrm{GeV})$ for 0,45 , and $90^{\circ}$ 
It should also be noted that the D meson production cross section not only narrows with increasing laboratory angle, but its maximum becomes lower and shifts toward lower particle momenta (Fig. 4). Thus, a $2 \mathrm{GeV} / \mathrm{c}$ D meson can be registered with $\mathrm{dN} / \mathrm{dp}$ on a level of $10-5$ at $90^{\circ}, 2 \cdot 10-4$ at $45^{\circ}$, and $4.5 \cdot 10-3$ at $0^{\circ}$, which gives at least two orders of magnitude growth toward the zero angle.

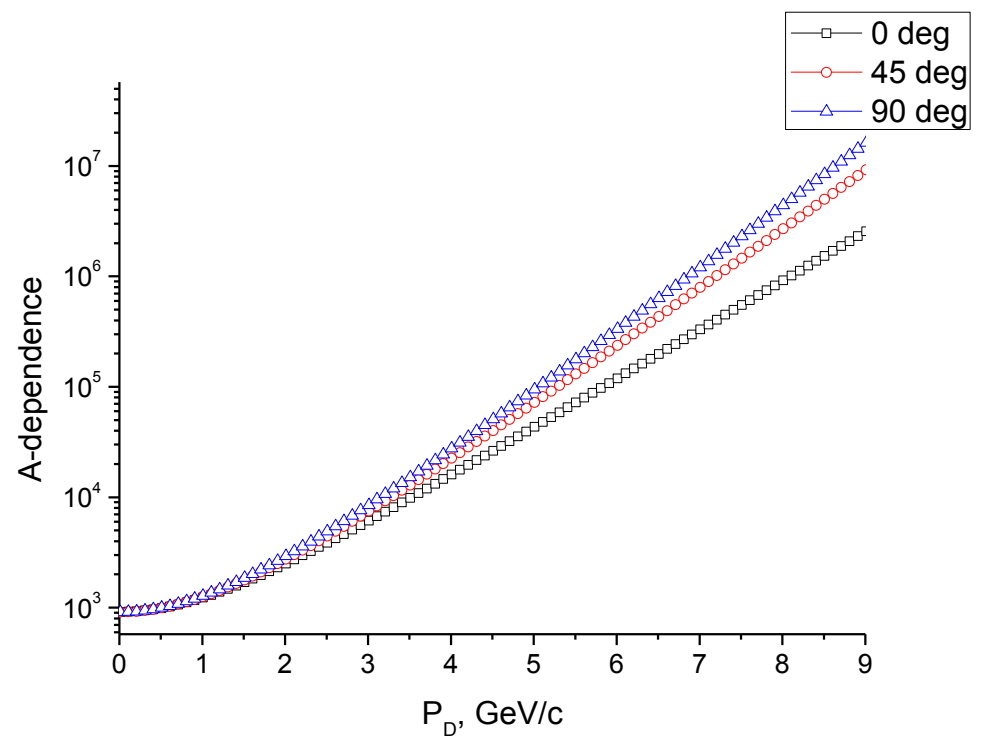

Figure 5. A-dependence of the cross section as a function of particle momentum in $\mathrm{Au}-\mathrm{Au}$ collisions at $2 \mathrm{GeV} / \mathrm{c}$ for 0,45 , and $90^{\circ}$

Let us consider the role of collectivity in the $\mathrm{D}$ meson production in $\mathrm{Au}-\mathrm{Au}$ collisions. Fig. 5 illustrates the character of the A-dependence in the cross section with increasing particle momentum for three different angles of particle production in the laboratory frame. It can be seen from Fig. 5 that the A-dependence is a fast growing function of the particle momentum. If we increase the momentum of the detected particle by $1 \mathrm{GeV} / \mathrm{c}$, the Adependence value (and therefore, the particle production cross section) increases several times. Moreover, the sharper it is, the larger the angle. A D meson with the same momentum detected at different angles corresponds to the different reaction "collectivity" (the A dependence value). 


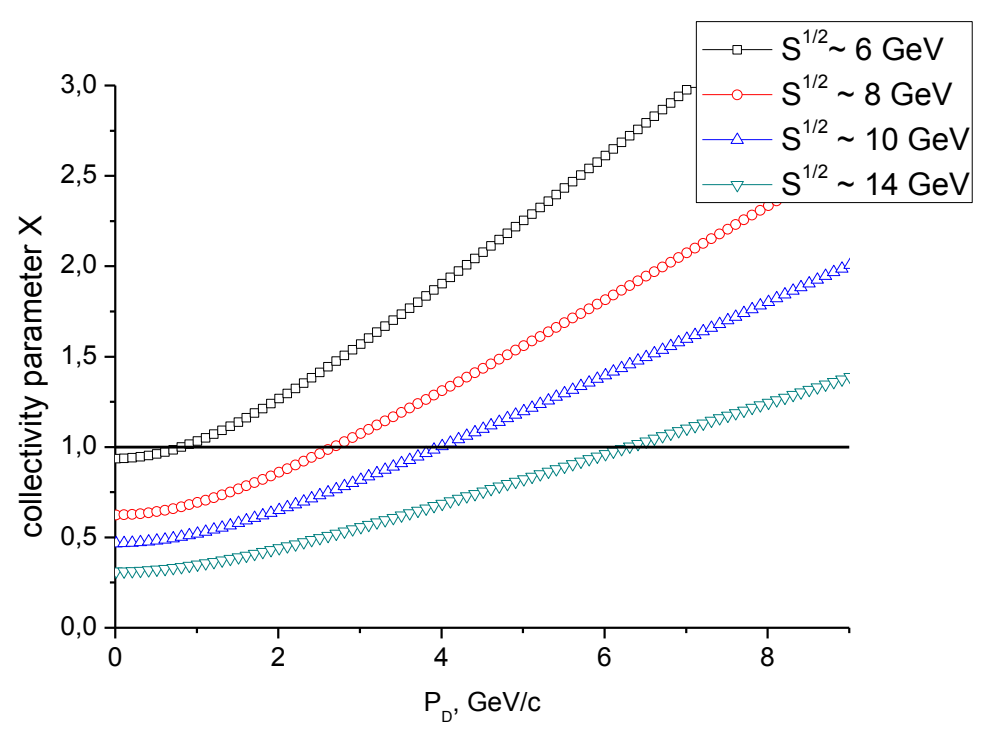

Figure 6. D meson production at $90^{\circ}$ in $\mathrm{Au}-\mathrm{Au}$ collisions

The "collectivity" effect is illustrated in Fig. 6. In this figure, the fraction of fourmomentum (or number of nucleons) participating in the reaction (X) is plotted as a function of $\mathrm{D}$ meson momentum. Horizontal line $\mathrm{X}=1$ shows the boundary of single-nucleon interactions for both colliding nuclei (below $X=1$ the particle can be produced in a singlenucleon interaction, while above $\mathrm{X}=1$ more than one nucleon from each colliding nucleus is required).

It can be seen that while in $\mathrm{Au}-\mathrm{Au}$ collisions at $\sim 6 \mathrm{GeV} / \mathrm{c}$ collective (with respect to both colliding nuclei) phenomena occur for rather high $\mathrm{D}$ meson momenta $(6 \mathrm{GeV} / \mathrm{c}$ and higher), moderate collider energies $(\sim 2 \mathrm{GeV} / \mathrm{c} \mathrm{Au})$ require multinucleon interaction of both nuclei to produce $\mathrm{D}$ mesons at less than $1 \mathrm{GeV} / \mathrm{c}$.

It was shown above that multi-nucleon processes of $\mathrm{D}$ meson production are experimentally measurable in a wide range of collision energies covered by the NICA collider.

We point out that the kinematic parameters at which collective effects are manifested can thus be optimized from the point of view of experimental feasibility and the specific features of the installations at the NICA accelerator complex.

\section{References}

1. A. A. Baldin, Phys. Part. Nucl. 56(3), 385 (1993)

2. A. M. Baldin and A. A. Baldin, Phys. Part. Nucl. 29(3), 577 (1998)

3. A. A. Baldin, E.G. Baldina, in Selected Papers of the Seminar (2000-2005) Symmetries and Integrable Systems, Ed. A. N. Sissakian, V.1 (JINR, Dubna, 2006) 41

4. A. A. Baldin et al., JINR Rapid Comm. 3(92), 20 (1992)

5. A.A. Baldin, E.G. Baldina, E.N. Kladnitskaya, and O.V. Rogachevsky, Phys. Part. Nucl. Lett. 121, 7 (2004)

6. E. G. Baldina and A. A. Baldin, EPJ Web of Conferences "Baldin ISHEPP XXIIII" 138, 05001 (2017)

7. The ALICE Collaboration, Int. J. Mod. Phys. A 29(24), 1430044 (2014) 